\section{A Sampling Theorem for Shift-Invariant Subspace}

Wen Chen and Shuichi Itoh

\begin{abstract}
A sampling theorem for regular sampling in shift invarian subspaces is established. The sufficient-necessary condition for which it holds is found. Then, the theorem is modified to the shift sampling in shiftinvariant subspaces by using the Zak transform. Finally, some examples are presented to show the generality of the theorem.
\end{abstract}

Index Terms-Fourier transform, generating function, sampling, shiftinvariant subspace, Zak transform.

\section{INTRODUCTION AND NOTATIONS}

A fundamental question in signal processing is how to represent a signal in terms of a discrete sequence. Shannon's popular sampling theorem states that finite energy band-limited signals are completely characterized by their samples values. Realizing that the Shannon interpolating function $\operatorname{sinc}(t)=\sin (t) / t$ is in fact a scaling function of an MRA, Walter [18] found a sampling theorem for a class of wavelet subspaces.

Suppose $\varphi(t)$ is a continuous orthonormal scaling function of an MRA $\left\{V_{m}\right\}_{m \in Z}$ such that $|\varphi(t)| \leq O\left((1+|t|)^{-1-\varepsilon}\right)$ for some $\varepsilon>0$. Let $\hat{\varphi}^{*}(\omega)=\sum_{n} \varphi(n) e^{-i n \omega}$. Walter showed that there is an $S(t) \in V_{0}$ such that

$$
f(t)=\sum_{n \in Z} f(n) S(t-n)
$$

holds for any $f(t) \in V_{0}$ if $\hat{\varphi}^{*}(\omega) \neq 0$. Following Walter's [18] work, Janssen [11] studied the shift sampling case by using the Zak-transform. Xia and Zhang [21] discussed the so-called sampling property $(S(t)=\varphi(t))$. Walter [19], Xia [20], and Chen-Itoh [8], [9] studied the more general case "oversampling." Chen et al. [5], [7], Chen and Itoh [6], Liu [12], and Liu and Walter [13] even studied irregular sampling in wavelet subspaces.

Furthermore, Aldroubi and Unser [1]-[3], [15] studied the sampling procedure in shift invariant subspaces (see Section II). They established a more comprehensive sampling theory for shift-invariant subspaces. One of their important result states that when $\varphi(t)(\epsilon$ $L^{2}(R)$ ) is a generating function, the orthogonal projection $g_{p}(t)$ of a function $g(t) \in L^{2}(R)$ on the shift-invariant subspace $V_{0}(\varphi)$ is given by

$$
g_{p}(t)=\sum_{n \in Z}\langle g(\cdot), \tilde{\varphi}(\cdot-n)\rangle \varphi(t-n)
$$

where $\{\tilde{\varphi}(t-n)\}_{n}$ is the biorthogonal basis of $\{\varphi(t-n)\}_{n}$ in $V_{0}(\varphi)$, and $\langle\cdot, \cdot\rangle$ is the $L^{2}(R)$-inner product. They then found that the $\varphi(t)$ can be replaced by an interpolating generating function $S(t)$ if $\varphi(t) \in L^{1}(R) \cap L^{2}(R), \sum_{k} \hat{\varphi}(\omega+2 k \pi) \neq 0$, and the Fourier transform $\hat{\varphi}(\omega)$ of $\varphi(t)$ satisfies $|\hat{\varphi}(\omega)| \leq O\left((1+|\omega|)^{-1-\varepsilon}\right)$ for some $\varepsilon>0$ (see [3, Prop 7]). In fact, these constraints are related to those of Walter sampling theorem due to the fact $\sum_{k} \hat{\varphi}(\omega+2 k \pi)=\hat{\varphi}^{*}(-\omega)$ in some sense (see the Appendix).

Manuscript received April 16, 1997; revised March 20, 1998. The associate editor coordinating the review of this paper and approving it for publication was Dr. Akram Aldroubi.

The authors are with the Department of Information Network Science, Graduate School of Information Systems, University of Electro-Communications, Tokyo, Japan (e-mail: wchen@bach.net.is.uec.ac.jp).

Publisher Item Identifier S 1053-587X(98)07083-4.
Our purpose in this correspondence is to find a weaker constraint on the generating function $\varphi(t)$ such that a formula similar to (1) [or (2) with interpolating generating function $S(t)$ instead of $\varphi(t)$ ] holds for any function $f(t)$ in the shift-invariant subspaces $V_{0}(\varphi)$. In fact, we find a condition for (1) that is sufficient and necessary. In this way, we are able to remove the continuity and regularity constraints imposed on the generating function $\varphi(t)$ by Walter [18] or imposed on its Fourier transform $\hat{\varphi}(\omega)$ by Aldroubi and Unser [3]. We also make a case to show the generality of our result in Section III.

We now introduce some notations used in this correspondence. For a measurable subset $E \subset R,|E|$ denotes the measure of $E$. For a measurable function $f(t)$, we write

$$
\begin{aligned}
\|f(t)\|_{0} & =\sup _{|E|=0} \inf _{R \ominus E}|f(t)| \\
\|f(t)\|_{\infty} & =\inf _{|E|=0} \sup _{R \ominus E}|f(t)| \\
\chi_{E}(t) & = \begin{cases}1 & t \in E \\
0 & \text { otherwise. }\end{cases}
\end{aligned}
$$

\section{SAMPLING THEOREM}

Suppose an $L^{2}(R)$ function $\varphi(t)$ is such that the sampling $\{\varphi(n)\}_{n}$ makes sense and $\{\varphi(n)\}_{n} \in l^{2}$. Then, the series $\sum_{n} \varphi(n) e^{-i n \omega}$ converges to an $L^{2}[0,2 \pi]$ function $\hat{\varphi}^{*}(\omega)$ in $L^{2}[0,2 \pi]$ sense. Let us now consider the shift-invariant subspace sequence $\left\{V_{j}(\varphi)\right\}_{j}$ generated by $\varphi(t)$

$$
V_{j}(\varphi)=\left\{\sum_{k} c_{k} \varphi\left(2^{j} t-k\right):\left\{c_{k}\right\}_{k} \in l^{2}\right\} \subset L^{2}(R) .
$$

For $f(t)=\sum_{k} c_{k} \varphi(t-k) \in V_{0}(\varphi)$, we let $f(n)=\sum_{k} c_{k} \varphi(n-k)$. Then, $\{f(n)\}_{n}\left(\in l^{\infty}\right)$ is well defined since $\left\{c_{k}\right\}_{k}$ and $\{\varphi(k)\}_{k}$ are both $l^{2}$ sequences. In fact, $f(n)$ as the Fourier coefficients of the $L^{1}[0,2 \pi]$ function $\hat{\varphi}^{*}(\omega) \sum_{k} c_{k} e^{-i k \omega}$ tends to 0 at infinity by the Riemann-Lebesgue Lemma. Generally, $\{\varphi(t-k)\}_{k}$ may not be a Riesz basis of $V_{0}(\varphi)$. It is shown that $\{\varphi(t-k)\}_{k}$ is a Riesz basis of $V_{0}(\varphi)$ if and only if

$$
0<\left\|G_{\varphi}(\omega)\right\|_{0} \leq\left\|G_{\varphi}(\omega)\right\|_{\infty}<\infty
$$

holds, where $G_{\varphi}(\omega)=\left(\sum_{k}|\hat{\varphi}(\omega+2 k \pi)|^{2}\right)^{1 / 2}$, and $\hat{\varphi}(\omega)$ is the Fourier transform of $\varphi(t)$ defined by $\hat{\varphi}(\omega)=\int_{R} \varphi(t) e^{-i \omega t} d t$. If $\varphi(t)$ satisfies (7), it is called a generating function (see [3]).

Theorem 1: Suppose $\varphi(t)\left(\in L^{2}(R)\right)$ is a generating function such that the sampling $\{\varphi(n)\}_{n}$ makes sense, and $\{\varphi(n)\}_{n} \in l^{2}$. Then, there is an $S(t) \in V_{0}(\varphi)$ such that

$$
f(t)=\sum_{n} f(n) S(t-n), \quad \text { for } f(t) \in V_{0}(\varphi)
$$

holds in the $L^{2}(R)$ sense if and only if

$$
\frac{1}{\hat{\varphi}^{*}(\omega)} \in L^{2}[0,2 \pi]
$$

holds. In this case, $\hat{S}(\omega)=\hat{\varphi}(\omega) / \hat{\varphi}^{*}(\omega)$ holds for a.e. $\omega \in R$.

Proof:

Step 1-Sufficiency: Assume $1 / \hat{\varphi}^{*}(\omega) \in L^{2}[0,2 \pi]$. Then, $\hat{\varphi}^{*}(\omega) \neq 0$ holds for a.e. $\omega \in R$, and there is a $\left\{c_{k}\right\}_{k} \in l^{2}$ such that

$$
\frac{1}{\hat{\varphi}^{*}(\omega)}=\sum_{k} c_{k} e^{i k \omega}
$$


holds in the $L^{2}[0,2 \pi]$ sense. Let $F(\omega)=\hat{\varphi}(\omega) / \hat{\varphi}^{*}(\omega)$. Then

$$
\begin{aligned}
\int_{R}|F(\omega)|^{2} & =\int_{R}\left|\frac{\hat{\varphi}(\omega)}{\hat{\varphi}^{*}(\omega)}\right|^{2} \\
& =\int_{0}^{2 \pi} \frac{\sum_{n}|\hat{\varphi}(\omega+2 n \pi)|^{2}}{\left|\hat{\varphi}^{*}(\omega)\right|^{2}} \\
& \leq\left\|G_{\varphi}(\omega)\right\|_{\infty}^{2} \int_{0}^{2 \pi} \frac{1}{\left|\hat{\varphi}^{*}(\omega)\right|^{2}} .
\end{aligned}
$$

It is easy to see $F(\omega) \in L^{2}(R)$ due to (7). Hence, we can take the Fourier inverse of $F(\omega)$ in $L^{2}(R)$ denoted by $S(t)$, i.e., we derive

or

$$
\hat{S}(\omega)=\frac{\hat{\varphi}(\omega)}{\hat{\varphi}^{*}(\omega)}
$$

$$
\hat{\varphi}(\omega)=\hat{S}(\omega) \hat{\varphi}^{*}(\omega) .
$$

Take inverse Fourier transform on both sides of (11) and refer to (10)

$$
S(t)=\sum_{k} c_{k} \varphi(t-k) \text {. }
$$

Formula (13) implies $S(t) \in V_{0}(\varphi)$ [due to the fact that $\{\varphi(t-k)\}_{k}$ is a Riesz basis of $V_{0}(\varphi)$ ]. For any $f(t) \in V_{0}(\varphi)$, there is a $\left\{a_{k}\right\}_{k} \in l^{2}$ such that $f(t)=\sum_{k} a_{k} \varphi(t-k)$. Then

$$
\begin{aligned}
\hat{f}(\omega) & =\hat{\varphi}(\omega) \sum_{k} a_{k} e^{-i k \omega} \\
& =\left(\hat{\varphi}^{*}(\omega) \sum_{k} a_{k} e^{-i k \omega}\right) \hat{S}(\omega) .
\end{aligned}
$$

Therefore, $f(t)=\sum_{k} f(k) S(t-k)$.

Step 2-Necessity: On the contrary, if there is an $S(t) \in V_{0}(\varphi)$ such that (8) holds in the $L^{2}(R)$ sense, then

$$
\varphi(t)=\sum_{n} \varphi(n) S(t-n)
$$

holds in the $L^{2}(R)$ sense. By taking the Fourier transform on both sides of (16), we obtain

$$
\hat{\varphi}(\omega)=\hat{\varphi}^{*}(\omega) \hat{S}(\omega) .
$$

Equation (17) implies that $\operatorname{supp} \hat{\varphi}(\omega) \subset \operatorname{supp} \hat{\varphi}^{*}(\omega)$ holds for a.e. $\omega \in R$, i.e., $\operatorname{supp} \hat{\varphi}(\omega+2 k \pi) \subset \operatorname{supp} \hat{\varphi}^{*}(\omega)$ holds for all $k \in Z$ and for a.e. $\omega \in R$ because $\hat{\varphi}^{*}(\omega)$ is $2 \pi$ periodic. Meanwhile

$$
\bigcup_{k} \operatorname{supp} \hat{\varphi}(\omega+2 k \pi)=R
$$

holds except for a zero measure subset of $R$. Otherwise, there is a measurable subset $\delta$ with measure $|\delta| \neq 0$ such that

$$
\delta \subset R \ominus \bigcup_{k} \operatorname{supp} \hat{\varphi}(\omega+2 k \pi) .
$$

Then, $\hat{\varphi}(\omega+2 k \pi)=0$ holds for any $\omega \in \delta$ and for all $k \in Z$. Hence

$$
G_{\varphi}(\omega)=\left(\sum_{k}|\hat{\varphi}(\omega+2 k \pi)|^{2}\right)^{1 / 2}=0
$$

holds for any $\omega \in \delta$. However, $G_{\varphi}(\omega) \neq 0$ holds for a.e. $\omega \in R$. It forces (18) to hold for a.e. $\omega \in R$. Therefore

$$
\operatorname{supp} \hat{\varphi}^{*}(\omega) \supset \bigcup_{k} \operatorname{supp} \hat{\varphi}(\omega+2 k \pi)=
$$

holds for a.e. $\omega \in R$, i.e., $\hat{\varphi}^{*}(\omega) \neq 0$ for a.e. $\omega \in R$. Formula (17) is now rewritten to be

$$
\frac{\hat{\varphi}(\omega)}{\hat{\varphi}^{*}(\omega)}=\hat{S}(\omega)
$$

Since $\hat{S}(\omega) \in L^{2}(R)$ [due to $S(\omega) \in L^{2}(R)$ ], we derive

$$
\begin{aligned}
\infty>\int_{R}\left|\frac{\hat{\varphi}(\omega)}{\hat{\varphi}^{*}(\omega)}\right|^{2} & =\int_{0}^{2 \pi} \frac{\sum_{k}|\hat{\varphi}(\omega+2 k \pi)|^{2}}{\left|\hat{\varphi}^{*}(\omega)\right|^{2}} \\
& \geq\left\|G_{\varphi}^{2}(\omega)\right\|_{0} \int_{0}^{2 \pi}\left|\frac{1}{\hat{\varphi}^{*}(\omega)}\right|^{2} .
\end{aligned}
$$

From (7) and (23), we conclude that $1 / \hat{\varphi}^{*}(\omega) \in L^{2}[0,2 \pi]$ holds. This completes the proof.

If $\varphi(t)$ satisfies the conditions of the Walter sampling theorem or the proposition of Aldroubi and Unser, there must be a constant $C \geq 1$ such that $C^{-1} \leq\left|\hat{\varphi}^{*}(\omega)\right| \leq C$. Obviously, $1 / \hat{\varphi}^{*}(\omega) \in$ $L^{\infty}[0,2 \pi] \subset L^{2}[0,2 \pi]$. Therefore, the Walter sampling theorem and the Aldroubi and Unser proposition can be obtained as a corollary of our theorem (refer to Examples 1-3). A related problem is the study of truncation error and aliasing error. We do not estimate them here and refer to Walter, Unser and Daubechies [16] and Chen and Itoh [8], [9].

As done by Janssen [11] for Walter's sampling theorem, Chen et al. [7] for the irregular sampling theorem, and Chen and Itoh [8] for the oversampling theorem, the shift-sampling theorem for shift-invariant subspace can be obtained by using the Zak transform. Suppose $\varphi(t)$ $\left(\in L^{2}(R)\right)$ is such that the sampling $\{\varphi(\sigma+n)\}_{n}$ makes sense, and $\{\varphi(\sigma+n)\}_{n} \in l^{2}$ for some $\sigma \in[0,1)$. Then, the Zak transform $Z_{\varphi}(\sigma, \omega)$ of $\varphi(t)$ is defined by

$$
Z_{\varphi}(\sigma, \omega)=\sum_{n} \varphi(n+\sigma) e^{-i n \omega} .
$$

A generating function $\varphi(t)$ may not satisfy $1 / \hat{\varphi}^{*}(\omega) \in L^{2}[0,2 \pi]$ but may satisfy $1 / Z_{\varphi}(\sigma, \omega) \in L^{2}[0,2 \pi]$ for some $\sigma \in[0,1)$. Then, it can be dealt with by the shift-sampling theorem (see Example 4). We now present the shift-sampling theorem without proof (since it is very close to the previous).

Theorem 2: Suppose $\varphi(t)\left(\in L^{2}(R)\right)$ is a generating function such that the sampling $\{\varphi(n+\sigma)\}_{n}$ makes sense, and $\{\varphi(n+\sigma)\}_{n} \in$ $l^{2}$ for some $\sigma \in[0,1)$. Then, there is an $S_{\sigma}(t) \in V_{0}(\varphi)$ such that

$$
f(t)=\sum_{n} f(n+\sigma) S_{\sigma}(t-n), \quad \text { for } f(t) \in V_{0}(\varphi)
$$

holds in the $L^{2}(R)$ sense if and only if

$$
\frac{1}{Z_{\varphi}(\sigma, \omega)} \in L^{2}[0,2 \pi]
$$

holds. In this case, $\hat{S}(\omega)=\hat{\varphi}(\omega) / Z_{\varphi}(\sigma, \omega)$ holds for a.e. $\omega \in R$.

\section{SOME EXAMPLES}

Since the Haar function is not continuous and Shannon's sinc function is not regular enough, they can not be covered by Walter's sampling theorem. Since the Fourier transform of the Haar function is not regular enough and the Fourier transform of the Shannon function is not continuous, they are covered by [3, prop. 7], although we should note that there is no such restriction for the more general sampling theorems presented in Aldroubi and Unser's papers. Both functions are covered by our sampling theorem (see Examples 1 and 2).

Example 1: Haar function $\varphi(t)=\chi_{[0,1)}$. The piecewise continuity of $\varphi(t)$ implies that the sampling $\{\varphi(n)\}_{n}$ makes sense. $1 / \hat{\varphi}^{*}(\omega)=1 \in L^{2}[0,2 \pi]$ implies that our sampling theorem can be applied and $S(t)=\chi_{[0,1)}$. 
Example 2: Shannon function $\varphi(t)=\sin \pi t / \pi t$. The continuity of $\varphi(t)$ implies that the sampling $\{\varphi(n)\}_{n}$ makes sense. $1 / \hat{\varphi}^{*}(\omega)=$ $1 \in L^{2}[0,2 \pi]$ implies that our sampling theorem can be applied, and $S(t)=\sin \pi t / \pi t$.

The following Example 3 shows that there exists a generating function $\varphi(t)$ such that $\hat{\varphi}^{*}(\omega) \rightarrow 0$ as $\omega$ tends to a point $\omega_{0}$ (a.e.), but $1 / \hat{\varphi}^{*}(\omega) \in L^{2}[0,2 \pi]$ holds. It implies that our sampling theorem is substantially more general than Walter sampling theorem.

Example 3: For a positive number $s<1 / 2$, take $\varphi(t)$ as the Fourier inverse of $\hat{\varphi}(\omega)$ defined by

$$
\hat{\varphi}(\omega)= \begin{cases}-1, & \omega \in[-4 \pi,-2 \pi) \\ 1, & \omega \in[-2 \pi, 0) \\ \omega^{s}, & \omega \in[0,2 \pi) \\ 0, & \text { otherwise. }\end{cases}
$$

Then, $G_{\varphi}(\omega) \chi_{[0,2 \pi)}=\left(2+\omega^{2 s}\right)^{1 / 2}$. Obviously,

$$
\sqrt{2} \leq G_{\varphi}(\omega) \leq\left(2+(2 \pi)^{2 s}\right)^{1 / 2} .
$$

Therefore, $\varphi(t)$ is a generating function. The fact $\hat{\varphi}(\omega) \in L^{1}(R)$ implies that $\varphi(x)$ is continuous. Then, the sampling $\{\varphi(n)\}_{n}$ makes sense. Since $\hat{\varphi}^{*}(\omega)=\sum_{k} \hat{\varphi}(\omega+2 k \pi)$ in $L^{2}[0,2 \pi]$ (see the Appendix), we derive $\hat{\varphi}^{*}(\omega) \chi_{[0,2 \pi)}=\omega^{s}$. However, $\hat{\varphi}^{*}(\omega) \rightarrow 0$ as $\omega \rightarrow 0^{+}$(a.e.). Hence, neither Walter's sampling theorem nor Aldroubi and Unser's Proposition can be applied to deal with the $\varphi(t)$ [since both of them require the condition $C^{-1} \leq \hat{\varphi}^{*}(\omega) \leq C$ for some $C \geq 1]$. However, $\omega^{-s} \in L^{2}[0,2 \pi]$ implies that our sampling theorem is available. The $\hat{S}(\omega)$ is given by

$$
\hat{S}(\omega)= \begin{cases}-(\omega+4 \pi)^{-s}, & \omega \in[-4 \pi,-2 \pi) \\ (\omega+2 \pi)^{-s}, & \omega \in[-2 \pi, 0) \\ 1, & \omega \in[0,2 \pi) \\ 0, & \text { otherwise. }\end{cases}
$$

The following Example 4 (taken by Janssen [11]) shows the usefulness of shift-sampling theorem. It is also very interesting to find some works on centered spline interpolating in Aldroubi et al. [4] and Unser et al. [14].

Example 4: B-spline of order 2 scaling function

$$
\begin{aligned}
N_{2}(t)= & \frac{t^{2}}{2} \chi_{[0,1)}(t)+\frac{6 t-2 t^{2}-3}{2} \chi_{[1,2)}(t) \\
& +\frac{(3-t)^{2}}{2} \chi_{[2,3)}(t) .
\end{aligned}
$$

$N_{2}(t)$ is a generating function (see Chui [10]). $\hat{N}_{2}^{*}(\omega)=e^{i \omega}\left(e^{i \omega}+\right.$ 1) $/ 2$ implies that $1 / N_{2}^{*}(\omega)=2 / e^{i \omega}\left(e^{i \omega}+1\right)$ is not an $L^{2}[0,2 \pi]$ function. However, $1 / Z_{N_{2}}(1 / 2, \omega)=8 /\left(1+6 e^{i \omega}+e^{2 i \omega}\right) \in$ $L^{2}[0,2 \pi]$ implies that the shift-sampling theorem is available. The $\hat{S}_{1 / 2}(\omega)$ is given by

$$
\hat{S}_{1 / 2}(\omega)=8\left(\frac{1-e^{-i \omega}}{i \omega}\right)^{3} /\left(1+6 e^{i \omega}+e^{2 i \omega}\right) .
$$

\section{APPENDIX}

Claim-A Variation of Poission's Summation Formula: Suppose a measurable function $K(x)$ is such that $\sum_{n} K(x+2 n \pi)$ absolutely converges in $L^{2}[0,2 \pi]$. Then, $\sum_{n} K(x+2 n \pi)=\sum_{n} \hat{K}(n) e^{i n x}$ in $L^{2}[0,2 \pi]$.

Proof: Obviously, $\sum_{n} K(x+2 n \pi)$ absolutely converges in $L^{1}[0,2 \pi]$. Hence, $\int_{R}|K(x)| d x=\int_{0}^{2 \pi} \sum_{n}|K(x+2 n \pi)| d x<$ $\infty$, i.e., $K(x) \in L^{1}(R)$. This implies that $\hat{K}(\omega)$ is continuous [therefore, $\hat{K}(n)$ is well defined]. Let $g(x)=\sum_{n} K(x+2 n \pi)$ in $L^{2}[0,2 \pi]$. Then, $g(x)=\sum_{n} c_{n}(g) e^{i n x}$ in $L^{2}[0,2 \pi]$ with the Fourier coefficients $c_{n}(g)=\int_{0}^{2 \pi} g(x) e^{-i n x}=\hat{K}(n)$. Finally, $g(x)=\sum_{n} \hat{K}(n) e^{i n x}$ in $L^{2}[0,2 \pi]$. This completes the proof.
In Aldroubi and Unser [3], we let $\tilde{\tilde{\varphi}}(t)$ be the Fourier inverse of $\hat{\varphi}(\omega)$, and let $K(\omega)=\hat{\varphi}(\omega)$. Then, $\tilde{\tilde{\varphi}}(t)=\varphi(t)$ in $L^{2}(R)$, and $\sum_{n} \hat{\varphi}(\omega+2 n \pi)=\hat{\tilde{\varphi}}^{*}(-\omega)$ in $L^{2}[0,2 \pi]$. However, the $\varphi(t)$ in Example 3 is exactly the Fourier inverse of $\hat{\varphi}(\omega)$.

\section{ACKNOWLEDGMENT}

The authors greatly appreciate the referees for their very good suggestions. They also thank Prof. T. Hashimoto, Dr. J. Shiki, Dr. Y. Zhou, and Dr. W. Zhen for their partial contributions.

\section{REFERENCES}

[1] A. Aldroubi and M. Unser, "Families of wavelet transforms in connection with Shannon sampling theory and the Gabor transform," in Wavelets: A Tutorial in Theory and Applications, C. K. Chui, Ed. New York: Academic, 1992, pp. 509-528.

[2] A. Aldroubi and M. Unser, "Families of multi-resolution and wavelet spaces with optimal properties," Numer. Funct. Anal. Optimiz., vol. 14, pp. 417-446, 1993.

[3] _ _ "Sampling procedures in function spaces and asymptotic equivalence with Shannon sampling theorem," Numer. Funct. Anal. Optimiz., vol. 15, pp. 1-21, 1994.

[4] A. Aldroubi, M. Unser, and M. Eden, "Cardinal spline filters: Stability and convergence to the ideal sinc interpolator," Signal Process., vol. 28, no. 2, pp. 127-138, 1992.

[5] W. Chen, S. Itoh, and J. Shiki, "Sampling theorem by wavelets for irregularly sampled signals," IEICE Trans. Fundamentals, vol. J79-A, no. 12, pp. 1941-1949, 1996.

[6] W. Chen and S. Itoh, "On irregular sampling in wavelet subspaces," IEICE Trans. Fundamentals, vol. E80-A, no. 7, pp. 1299-1307, 1997.

[7] W. Chen, S. Itoh, and J. Shiki, "Irregular sampling theorem for wavelet subspaces," IEEE Trans. Inform. Theory, vol. 44, pp. 1131-1142, 1998.

[8] W. Chen and S. Itoh, "Oversampling theorem for wavelet subspaces," IEICE Trans. Fundamentals, vol. E81-A, no. 1, pp. 131-138, 1998.

[9] _ _ "Signal reconstruction by scaling functions with oversampling property," in Proc. IEICE-SITA, 1997, vol. 20, pp. 745-748.

[10] C. K. Chui, "An introduction to wavelets," in Wavelet Analysis and Application. New York: Academic, 1992, vol. 1.

[11] A. J. E. M. Janssen, "The Zak-transform and sampling theorem for wavelet subspaces," IEEE Trans. Signal Processing, vol. 41, pp. 3360-3364, Dec. 1993.

[12] Y. Liu, "Irregular sampling for spline wavelet subspaces," IEEE Trans. Inform. Theory, vol. 42, pp. 623-627, Apr. 1996.

[13] Y. Liu and G. G. Walter, "Irregular sampling in wavelet subspaces," $J$. Fourier Anal. Application, vol. 2, no. 2, pp. 181-189, 1995.

[14] M. Unser, A. Aldroubi, and M. Eden, "Fast B-spline transforms for continuous image representation and interpolation," IEEE Trans. Pattern Anal. Machine Intell., vol. 13, pp. 277-285, Mar. 1991.

[15] M. Unser and A. Aldroubi, "A general sampling theorem for nonideal acquisition devices," IEEE Trans. Signal Processing, vol. 42, pp. 2915-1925, Nov. 1994.

[16] M. Unser and I. Daubechies, "On the approximation power of convolution-based least square versus interpolation," IEEE Trans. Signal Processing, vol. 45, pp. 1697-1711, July 1997.

[17] Y. Meyer, "Wavelets and iperators," in Cambridge Studies in Advanced Math. 37. Cambridge, U.K.: Cambridge Univ. Press, 1992.

[18] G. G. Walter, " A sampling theorem for wavelet subspace," IEEE Trans. Inform. Theory, vol. 38, pp. 881-884, Apr. 1992.

[19] _ "Wavelet subspaces with an oversampling property," Indaga. Math., vol. 4, no. 4, pp. 499-507, 1993.

[20] X.-G. Xia, "On orthogonal wavelets with the oversampling property," J. Fourier Anal. Applicat., vol. 2, pp. 193-199, 1994.

[21] X.-G. Xia and Z. Zhang, "On sampling theorem, wavelets, and wavelet transforms," IEEE Trans. Signal Processing, vol. 41, pp. 3524-3534, Dec. 1993 